\title{
MESENCHYMAL STEM CELLS AND THEIR SECRETOME - CANDIDATES FOR SAFE AND EFFECTIVE THERAPY FOR SYSTEMIC LUPUS ERYTHEMATOSUS
}

Małgorzata Popis ${ }^{1}$, Aneta Konwerska², Małgorzata Partyka², Maria Wieczorkiewicz ${ }^{3}$, Sylwia Ciesiółka², Katarzyna Stefańska², Julia Spaczyńska ${ }^{1}$, Afsaneh Golkar-Narenji ${ }^{4}$, Michal Jeseta $^{5,6}$, Dorota Bukowska ${ }^{7}$ Paul Mozdziak ${ }^{4,8}$, Marta Dyszkiewicz-Konwińska ${ }^{1,9}$

\begin{abstract}
More than 80 diseases are currently classified as autoimmune, with a rising prevalence throughout the world. Systemic lupus erythematosus (SLE) is classified as a systemic autoimmune disorder, but the exact pathogenesis of SLE remains elusive. Currently available treatment strategies offer only the possibility for disease remission making it essential to develop more effective and safer strategies for treatment. Recently MSCs are gaining attention as attractive therapeutic tools for autoimmune disease treatment. Special focus should be given to MSCs originated from perinatal tissues such as Wharton's jelly, as they present unique immunomodulatory properties and remarkably low immunogenicity. MSCs exert their immunomodulatory effects via direct cell-to-cell communication as well as in a paracrine manner, creating possibility to apply secretome of MSCs as an individual therapeutic tool. Although the secretome of MSCs has not yet been utilized in SLE treatment, its efficacy has been suggested in other disorders, such as multiple sclerosis or Alzheimer's disease. Regular administration of paracrine factors derived from MSCs could potentially effect in significant reduction of SLE symptoms and in maintenance of disease remission.
\end{abstract}

Running title: Mesenchymal stem cells as a therapy for systemic lupus erythematosus

Keywords: mesenchymal stem cells, secretome, conditioned media, exosomes, SLE

\footnotetext{
${ }^{1}$ Department of Anatomy, Poznań University of Medical Sciences, Poznań, Poland

${ }^{2}$ Department of Histology and Embryology, Poznań University of Medical Sciences, Poznań, Poland

${ }^{3}$ Department of Basic and Preclinical Sciences, Institute of Veterinary Medicine, Faculty of Biological and Veterinary Sciences, Nicolaus Copernicus University in Toruń, Toruń, Poland

${ }^{4}$ Prestage Department of Poultry Science, North Carolina State University, Raleigh, NC, USA

${ }^{5}$ Department of Obstetrics and Gynecology, University Hospital and Masaryk University, Brno, Czech Republic

${ }^{6}$ Department of Veterinary Sciences, Czech University of Life Sciences in Prague, Prague, Czech Republic

${ }^{7}$ Department of Clinical Sciences and Diagnostics, Institute of Veterinary Medicine, Nicolaus Copernicus University in Toruń, Toruń, Poland

${ }^{8}$ Physiology Graduate Program, North Carolina State University, Raleigh, NC, USA

${ }^{9}$ Department of Biomaterials and Experimental Dentistry, Poznań University of Medical Sciences, Poznań, Poland

*Correspondence: m.dyszkiewicz@ump.edu.pl

Full list of author information is available at the end of article
} 


\section{Introduction}

Autoimmune diseases are a heterogeneous group of more than 80 chronic conditions which affect about 7 to $10 \%$ of worldwide population [1]. Studies indicate that autoimmune incidence rate and prevalence have increased significantly over the last 30 years [2]. Their shared clinical feature is the presence of autoantibodies, raising immune response against patient's own tissues and causing chronic inflammation. There are two groups of autoimmune disorders, specified by the area of impact: organ-specific and systemic [3]. Etiopathogenesis of those illnesses remains unknown, although genetic and environmental, including hormonal factors are suggested to be involved in their development. Systemic lupus erythematosus (SLE) belongs to the group of systemic autoimmune diseases. It is a progressive condition characterized by periods of flare and remission. Immune complexes accumulate in organs over time and lead to their damage, with the kidneys being the most at risk. SLE can cause systemic organ failure and lead to death if untreated [4]. While it mostly affects women in reproductive age, its incidence and prevalence vary depending on ethnicity $[5,6]$. The last decades were abundant in development of multiple medicaments applicable to SLE. However, despite clearly improved patient survival, the treatment strategy still needs refinements as the use of currently available drugs is associated with a range of adverse side effects [7].

Recently, many studies have been conducted to explore the nature of mesenchymal stem cells (MSCs). It appears that MSCs exhibit great potential for effective and safe treatment of variety of diseases, especially autoimmune disorders such as systemic sclerosis [8] or type 1 diabetes mellitus [9]. MSCs possess immunomodulatory properties and tend to act both through cell-to-cell contact and by secretome, which includes soluble molecules and extracellular vesicles (EVs). The exosome, a carrier of nucleic acids, proteins and lipids, plays the most important role in the immunosuppressive properties of MSCs [10]. Numerous studies suggest that EVs are more useful than MSCs in therapy development because they do not carry risk of oncogenesis, and therapy tends to be less expensive $[11,12]$. It is clearly visible that application of MSCs secretome is gaining more and more attention in the scientific community. Nevertheless, there is still a need for research on application of MSCs secretome components in the treatment of SLE.

\section{Characteristics of systemic lupus erythematosus}

Further investigation of specific symptoms and markers of autoimmune diseases is vital in order to develop more appropriate treatment strategies. SLE is autoimmune disease which significantly affects the quality of life and negatively impacts the lifes- pan. In addition, there is currently no strategy that can provide a permanent cure of SLE and available drugs are burdened with adverse effects. It seems that dysfunction of MSCs may be involved in SLE pathogenesis, therefore indicating a novel possible therapeutic target.

\section{Epidemiology of SLE}

SLE is classified as a multifactorial, systemic, autoimmune disease with heterogenous spectrum of symptoms, mostly associated with chronic inflammation. The Lupus Foundation of America estimates that at least five million people worldwide suffer from of lupus [13]. The disease mostly manifests between the ages of 20 - 40. SLE affects women significantly more frequently than men, with a different ratio throughout populations, varying from $2: 1$ [14] to $14: 1$ [15]. Disease manifests most often in childbearing age $[5,6]$. Severity of symptoms may fluctuate during menstrual cycle and flares are more likely to occur during periods of rapid sex hormone changes like pregnancy. Such tendency can be explained by immunomodulatory capacity of sex hormones, as prolactin may alter immune tolerance disruption, while estrogen affects $\mathrm{T}$ cell and dendritic cell (DCs) regulation, leading to upregulation of IL-1, IL-4, IL-5, IL-10, TNF $\alpha$ and IFN- $\gamma$ and driving autoreactivity of B cells [16-19].

Higher frequency of SLE in females may be also triggered by genes located on sex chromosomes, highlighting the importance of genetic risk factors [6]. This was confirmed as numerous mutations are associated with SLE and may affect multiple components and mechanisms of the immune system response as DCs, B and T cells, immune complex processing or complement signaling. Risk loci include $\mathrm{C} 2$ and $\mathrm{C} 4$ genes of complement cascade, as well as DRB1 of MHC II and TNF $\alpha$ of MHC III. In turn, mutations in IRF5 or STAT4 are related to disruptions of IFNs signaling. Additionally, IRF5 mutation, affecting generation of Th17 response and IRF8, is implicated in repression of Th17 differentiation. Furthermore, defects of the PDRM-1 gene affect differentiation of plasma cells $[17,20]$.

However, the rate of monozygotic twin concordance, ranging between $24 \%$ and $69 \%$, supports the importance of environmental factor role in SLE development [21]. Ultraviolet light is included in this group, and exposure to this factor results in keratinocyte damage, DCs activation, and upregulation of proinflammatory cytokines, such as IFN- $\alpha$. Moreover, SLE onset may be correlated with application of certain medicaments such as TNF $\alpha$ inhibitors or IFNs $[17,19]$. It was suggested, as infection by some bacteria and viruses, particularly Epstein Barr Virus (EBV), may trigger SLE development. Sequences of some EBV proteins present homology to autoantibodies, and thus may undergo cross reaction with antigens, leading to development of autoreactivity [22]. 


\section{Pathogenesis of SLE}

Several mechanisms involved in development of autoimmunity SLE have been identified, including defects in clearance of apoptotic debris, break in tolerance of B cells and T cells, effecting in their autoreactivity, tissue inflammation and disruptions of immune regulation. Dysregulation of immune system leads to a situation where first autoimmune response drives the next one, of greater and more complexed intensity.

Pathological deficiencies in clearance of apoptotic debris are considered as a one of the core mechanisms of SLE development, as they promote the emergence of chronic inflammation [23]. Apoptosis may be induced by numerous mechanisms, such as UV light, DNA damage, cellular stress or cytokine withdrawal, and may act through extrinsic or intrinsic pathways. Finally, the phagocytic system recognizes the dying cell and effectively removes the apoptotic debris, including nucleoprotein complexes. Efficient clearance leads to enhancement of tolerogenic response and simplifies recognition of cellular components through anti-inflammatory action of cytokines, such as TGF- $\beta$ and IL-10, released by phagocytes. However, to enable efficient course of the process, membrane of a cell designated for apoptosis has to remain intact and capable to keep cellular components inside [24]. When apoptotic clearance is impaired, the cell may enter secondary necrosis and start to release signals causing disruption of immunotolerance [17]. In SLE macrophages present impairments of phagocytosis. Binding of opsonins, such as CRP or C1q, facilitates clearance of apoptotic cells. However, the level of CRP is reduced in SLE and thus may contribute to clearance impairment [25]. Inefficient digestion of apoptotic debris causes increased exposure of antigen presenting cells (APCs) for autoantigens. APCs are then able to enter germinal centers and present autoantigens to T cells and B cells, leading to loss of adaptive immune tolerance, induction of autoreactivity and production of autoantibodies. As a result, immune complexes (ICs) are formed, which stimulate plasmacytoid dendritic cells (pDCs) to generate and release cytokines, including IFN-1 [23]. IFN- $\gamma$ signaling may act in a feedback loop, leading to upregulation of MHC II expression and promotion of DCs maturation [17]. An example of this process is the action of IFN- $\alpha$, which presents an ability to stimulate expression of toll-like receptor 7 (TLR7) on myeloid dendritic cells (mDCs), and may induce IFN-1 production by pDCs through TLR7. SLE patients show increased expression of IFN-1 dependent genes [25].

Additionally, it was reported that, in SLE, an upregulation of MHC II expression is observed in DCs, which is suggested to drive the development of $\mathrm{T}$ cell autoreactivity in lymph nodes. Risk of HLA-D exposure for autoantigens and for presentation of au- toantigens to TCD4+ naive cells increases with disturbances of apoptotic debris clearance, infection or UV exposure. Cytokine storm leads to infiltration of phagocytes, including neutrophils, which can undergo NETosis in presence of IFN-1 and IL-1 $\beta$, IL-8, IL-17 and TNF. Through this specific process, neutrophils release extracellular traps (NETs), made up of DNA and RNA covered with histones, released in a form resembling a spider web. In samples taken from SLE patients, NETosis was observed. Further analysis revealed impairment of NETs degradation, as autoantibodies were opsonized by $\mathrm{C} 1 \mathrm{q}$, inhibiting DNase-1 functioning. Genetic material is opsonized by autoantibodies and forms ICs recognized by BCR cells or Fc $\gamma$ R on DCs, resulting in production of proinflammatory cytokines: IL-6, IL-8, IL-1 $\beta$, IL12 and TNF. NETosis occurring in SLE is suggested to be a major source of autoantibodies, driving activation of B cell and DCs. Such cascade of events leads to accumulation of IC in tissues and results in chronic inflammation of organs such as kidneys, joints, vasculature, skin, and nervous tissue, causing multiple organ failure and possibly resulting in death [25].

Contribution of the complement system to SLE development deserves special attention, because it is a major mechanism of innate immunity. This system consists of a number of inactive proteins circulating in blood. Interaction with ICs or cellular debris may induce cascades of enzymatic reactions driven by complement system components, as it was shown that its activity undergoes reduction during SLE flares [25]. Studies revealed that SLE is associated with genetic mutations of $\mathrm{C} 2$ and $\mathrm{C} 4$ components of the complement system [17]. Furthermore, it was suggested that ablation of C4 components may be involved in the loss of B cell tolerance [25].

Regarding B cell and T cell pathology in SLE, they should be considered as factors breaking immune tolerance, effecting in autoreactivity development. Self-reactive B cell generation is common. However, their general elimination is accomplished by mechanisms of central tolerance in bone marrow. Auto-responsive B cell removal is controlled also by peripheral mechanism, affecting their survival and inducing anergy. SLE patients serum presents upregulated levels of B lymphocyte stimulator (BLyS), known also as B-cell activating factor (BAFF) and it was suggested that it is involved in immune tolerance break and supports the survival of autoreactive B cells. Studies reveal that, in the course of SLE, both mechanisms are affected and naive B cell present defects in anergy. Somatic hypermutation in germinal centers (GCs) is typed as an alternative mechanism of autoreactivity perpetuation. Interaction of ThCD4+ cells with autoreactive B cell in GCs lead to class switching, differentiation to plasma cells and results in production of autoreactive IgG. Pathogenesis of this process may involve IFN- $\gamma$, TLR7, STAT1 
and IFN- $\gamma \mathrm{R}$, crucial for class switching mechanism [25]. Another mechanism potentially involved in the development of B cell auto-responsivity is molecular mimicry, in which infection followed by cell disruption and action of pro-inflammatory factors may lead to the development of cross reactivity, and stimulate development of an autoimmune response [22]. Additionally, disturbances in proportion of B cell subsets were noted in SLE, with increased frequency of plasma cells and memory cells, of which post-switched memory B cells showed to be less prone for immune suppression and able to undergo activation by cytokine cocktail, even in the absence of antigens or Th cells [17].

Interaction of $\mathrm{B}$ cells and $\mathrm{T}$ cells is essential for generation of IgG autoantibodies, high levels of which are present in the course of SLE. Class switching is also stimulated by $\mathrm{T}$ cell cytokines: TNF- $\alpha$, IFN- $\gamma$ and IL-10 [17]. Moreover, IFN- $\gamma$ R signaling stimulates the development of Th cells, resulting in IFN- $\gamma$, IL-5 and IL-6 upregulation in SLE. The above facts highlight the importance of $\mathrm{T}$ cell tolerance disturbances in SLE development. While mechanisms acting in the thymus are crucial for sustaining proper $\mathrm{T}$ cell responsivity, its control also occurs in the periphery and includes stimulation by Treg signaling, driving apoptosis, anergy or inhibition of auto-responsive T cells. Additionally, exposure of APCs HLA-D for autoantigens leads to their presentation to TCD4+ naive cells and forces autoreactivity development [25]. Disturbances of TCD4+ cause activation of its hypermetabolic state dominated by oxidation, mTORC1 activation, mitochondrial abnormalities and high glucose flux [26]. Moreover, upregulated levels of Th cells and their cytokines, such as IL-17, produced by Th17, correlate with SLE flares [17]. It has also been demonstrated that dysregulated proportion of ThCD4+ cells and Tregs may contribute to loss of tolerance in SLE [27]. Tregs play an important role in stimulation of peripheral anergy of autoreactive T cells. Functional impairment and reduction in the number of this subset of cells are observed in SLE [25]. Stimulation of Treg number may be influenced by tissue resident pDCs. However it was shown that number of these cells is downregulated in SLE. The study results demonstrate the ability of Tregs to suppress activation of Th and B cells, as well as Th cells proliferation and production of IFN- $\gamma$. Additionally, Treg presence in SLE increased susceptibility to undergo cell mediated death [17].

Several mediators of immune response deserve expanded discussion, as their role is crucial for perpetuation of SLE. IL-6 stimulates the growth of B cells, as well as differentiation and activation of both B cells and T cells. This cytokine also present an ability to activate other mediators of immune response. IFN- $\gamma$ produced by $\mathrm{B}$ cells controls the promotion of autoreactive GCs, as well as stimulates macrophage activation and the production of other cytokines. IFN- $\gamma$ can also be synthetized by Th1 cells, similarly to IL-2. Action of these agents is essential for stimulation of B cells to antibody production. Moreover, cytokines produced by Th2 cells, IL-4 and IL-5, together with action of IFN- $\gamma$, present an ability to stimulate leukocyte guidance to the lymph nodes. BLyS production is mainly provided by B cells, but they may be generated by other cells of the immune system as well and it has been shown that estradiol can stimulate its formation. BLyS present ability to mediate fate of B cells and stimulate their expansion, differentiation and production of antibodies. Moreover, it seems to play the role in the loss of B cell tolerance. BLyS presents the ability to stimulate B cells through three receptors, expression of which rises during SLE flares. Myeloid cells present in tissues, after induction by ICs, are capable of producing various cytokines, including IL- 6 and BLyS, thus promoting activation of B cells. IL-21, produced by Tfh cells, acts on B cells located in GCs, stimulating their expansion, class switching and differentiation to plasma cells. Additionally, this agent stimulates Tfh cell expansion, as well as Th17 differentiation $[17,25]$.

\section{MSCs deficiency in SLE}

Initially, it was suggested that disfunctions of hematopoietic cells can be the source of autoimmune disease emergence [28]. Currently, there is a strong scientific support of the theory that MSCs dysfunctions are an important factor of SLE development [29]. It was proven that MSCs derived from SLE patients present multiple structural and functional defects, comparing to MSCs derived from healthy cohort [30].

MSCs from SLE patients manifest disturbances in growth, proliferation and differentiation capacity. Moreover, they tend to be more prone to apoptosis and senescence, and present invalid cell migration [31,32]. More importantly, it was shown that MSCs taken from SLE patients present upregulated expression of 652 genes, while 1253 genes undergo downregulation [33].

Additionally, MSCs of SLE patients show abnormal cytokine production, impaired immunomodulatory functions, as well as disrupted ability to suppress $\mathrm{T}$ cell and B cell proliferation and differentiation of plasma cells [31,32]. In SLE, MSCs show upregulation of the Wnt/ $\beta$-catenin and p53 / p21 pathways, as well as downregulation of the Bcl-2 and indoleamine 2,3-dioxygenase (IDO) pathways, making them more susceptible to apoptosis, aging, and less effective at suppression of T lymphocyte activity. Moreover, MSCs isolated from SLE patients exhibit overexpression of the OAZ gene, causing olfactory 1 /early B cell factor-associated zinc finger protein upregulation, which may be involved in C-C motif chemokine ligand 2 (CCL2) downregulation, and result in disruption of MSCs abilities to suppress B-cell activity, proliferation and differentia- 
tion. Furthermore, MSCs from SLE patients showed increased production of reactive oxygen species, and it was noted that, in these cells, the mitochondrial antiviral signaling protein may act in a feedback loop, causing IFN- $\beta$ transcription and proinflammatory cytokine production [34].

Some findings also suggest that disfunctions of MSCs in SLE patients may result from disturbances in MSCs niche, as its environment is rich in pro-inflammatory mediators. It was shown that coculture of MSCs from healthy donors and B cells from SLE patients resulted in suppression of B cell proliferation and maturation, while incubation of MSCs from healthy donors with plasma from SLE patients caused inhibition of MSCs activity [32]. Additionally, disfunction of MSCs should be considered in regard to genetic implications of SLE. Genetic background of SLE may be associated with disfunctions of autologous MSCs. It is worth noting that, despite upregulation of Treg number, infusion of autologous MSCs to SLE patients did not bring any clinical benefits [35]. Yet, other studies bring evidence that allogenic transplantation of MSCs from healthy donors to SLE patients resulted in clinical remission or reduction of disease activity [36]. Such reports support a thesis that functionality of MSCs is affected in SLE and treatment with cells derived from healthy donors, lacking disturbances in MSCs niche, is effective and bring improvement in case of autoimmune conditions. The research also brings strong evidence that immunosuppressive potential of MSCs is strong and worth consideration regarding creation of a variety of therapies.

\section{Common methods of treatment}

SLE is a highly complex disease involving changes in the functioning of majority components of the immune system, including cooperation between the innate and adaptive immune response. Therefore, currently the treatment of SLE is carried out by administration of drugs acting on the immune pathways [17]. However, precise action of majority of these drugs is not completely understood. Substances accepted in the 1950's by the U.S. Food and Drug Administration as non-specific treatments include: aspirin, corticosteroids or hydroxychloroquine. Systemic immunosuppressants, such as cyclophosphamide, methotrexate and mycophenolate mofetil have also been introduced as part of the therapeutic strategy. First targeted biologic drug, Belimumab, was approved for use in 2011 [37]. However, the use of immunosuppressive drugs carries the risk of numerous side effects manifestation, such as infections, endocrine system dysregulation, vascular or heart failure and even secondary malignancy [38]. Additionally, SLE flares are still recorded during treatment, and unfortunately, some SLE patients are refractory to these drugs. Despite therapeutic introduction, the risk of premature death is two to five times higher than in case of general population [39].

Hence, invention of more effective and safer strategy for SLE treatment is essential. As MSCs impairment is observed in the course of SLE, they should be considered as a target for effective treatment. Studies on MSCs reveal that they present extremely broad effect on stimulation of immune mechanisms, especially towards immunotolerance upregulation

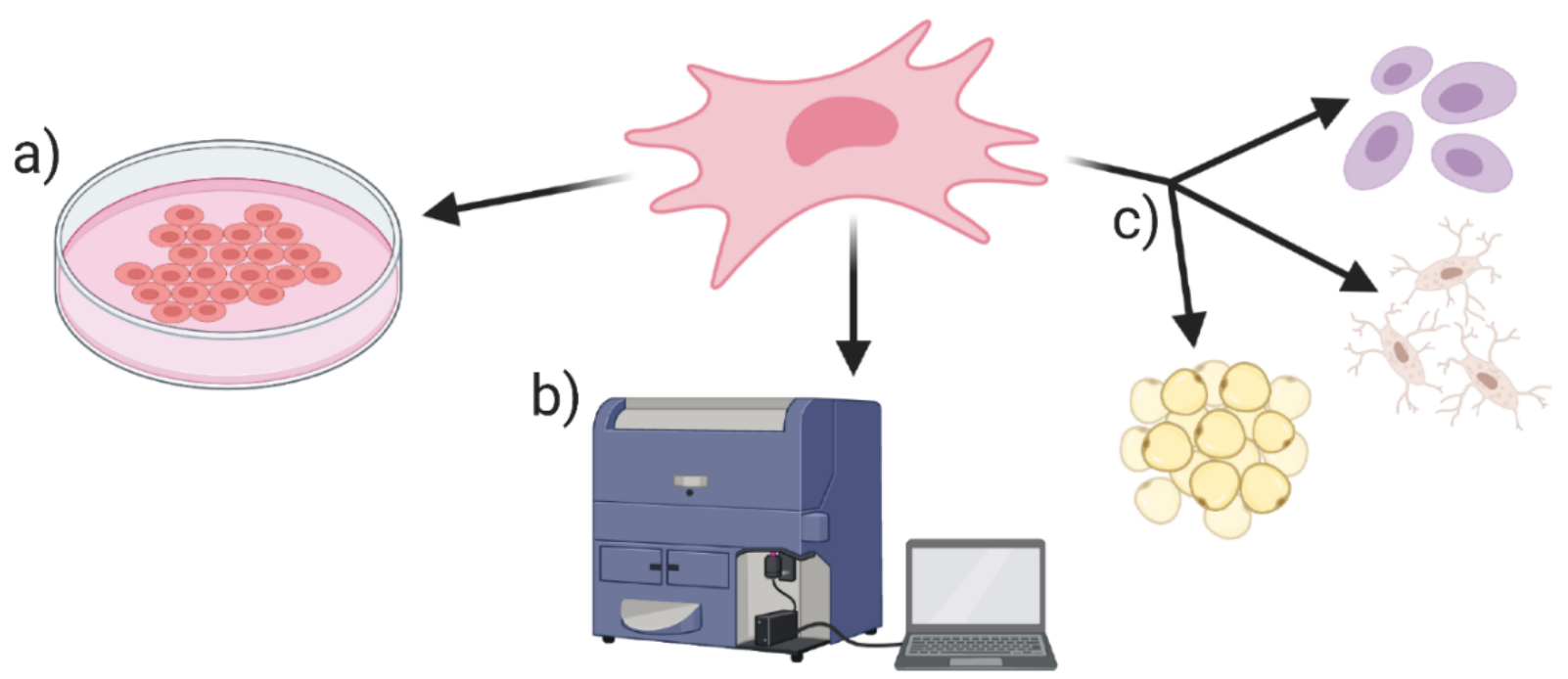

FIGURE 1 Identification of MSCs is based on following criteria, proposed and recommended by International Society for Cellular Therapy: a) cells must be plastic adherent during culture in standard conditions b) cells must exhibit presence of following markers: CD105, CD73, CD90 and reveal lack expression of following markers: CD45, CD34, HLA-DR, CD14 or CD11b, CD79a or CD19 c) cells must demonstrate the ability toward differentiation to adipocytes, chondroblasts, osteocytes upon culture in standard in vitro differentiating conditions. Created with BioRender.com 
[40], and therefore they are promising candidates for effective therapies against SLE, with a low risk of adverse side effects.

\section{Mesenchymal stem cells and their products - characteristic and properties}

Mesenchymal stem cells are currently one of the most studied subject in experimental medicine, given the fact that are easily obtainable and possess unique immunomodulatory properties, which is particularly relevant in treatment of autoimmune diseases. Depending on MSCs tissue of origin, the cell features may vary because of different microenvironment, known as the stem cell niche [41]. To improve standardization of studies on MSCs, the International Society for Cellular Therapy published "Minimal criteria for defining multipotent mesenchymal stromal cells", which recommends identification of MSCs based on following features: in standard culture conditions cells have to be adherent to plastic, cells have to express set of differentiation clusters on the membrane surface: CD105, CD73, CD90 and reveal lack of expression: CD45, CD34, HLA-DR, CD14 or CD11b, CD79a or CD19. Moreover, the cells must be able to differentiate into chondroblasts, osteoblasts and adipocytes in standard in vitro differentiating conditions (Fig. 1) [42].

MSCs were isolated for the first time from the bone marrow. MSCs were originally described as fibroblast-like cells forming colonies with a high level of replication capacity in in vitro conditions [43]. MSCs are successfully isolated from perinatal tissues, such as umbilical cord, placenta, amniotic fluid and amniotic membrane as well as from adult tissues like bone marrow, adipose tissue, dental pulp, spinal tissues, and peripheral blood vessels (Fig. 2) [44-49].

\section{MSCs derived from Wharton's jelly}

MSCs of perinatal origin exhibit unique properties of remarkably low immunogenicity and thus present significantly higher therapeutic potential than cells derived from adult tissues. An excellent example of such cell source is the umbilical cord (UC), with its outer layer constructed from amniotic epithelium, while the inner layer is closely connected with subamniotic Wharton's jelly (WJ). WJ is a mucoid connective tissue composed of collagen, glycoproteins and hyaluronic acid. Moreover, it is thought to present the highest density of MSCs among perinatal tissues. WJ is considered as an attractive source of MSCs because of its unique traits including: non-invasive collection, ease of sourcing, and lack of the ethical issues. Additionally, MSCs derived from WJ exhibit other noteworthy features as high proliferation rate, low immunogenicity, rapid self-renewal properties and most importantly, low risk of oncogenicity. Moreover, it should be emphasized that WJ is highly homogenous in regard of cell types, as it contains only MSCs in the form of myofibroblasts and their precursors, which is a desirable feature in context of its application within therapy [50]. UC structure also includes 2 veins and an artery with occasionally observed remnants of allantoic duct. Human Wharton's jelly mesenchymal stem cells (hWJ-MSCs) are most commonly isolated using mechanical extraction of explants or enzy-

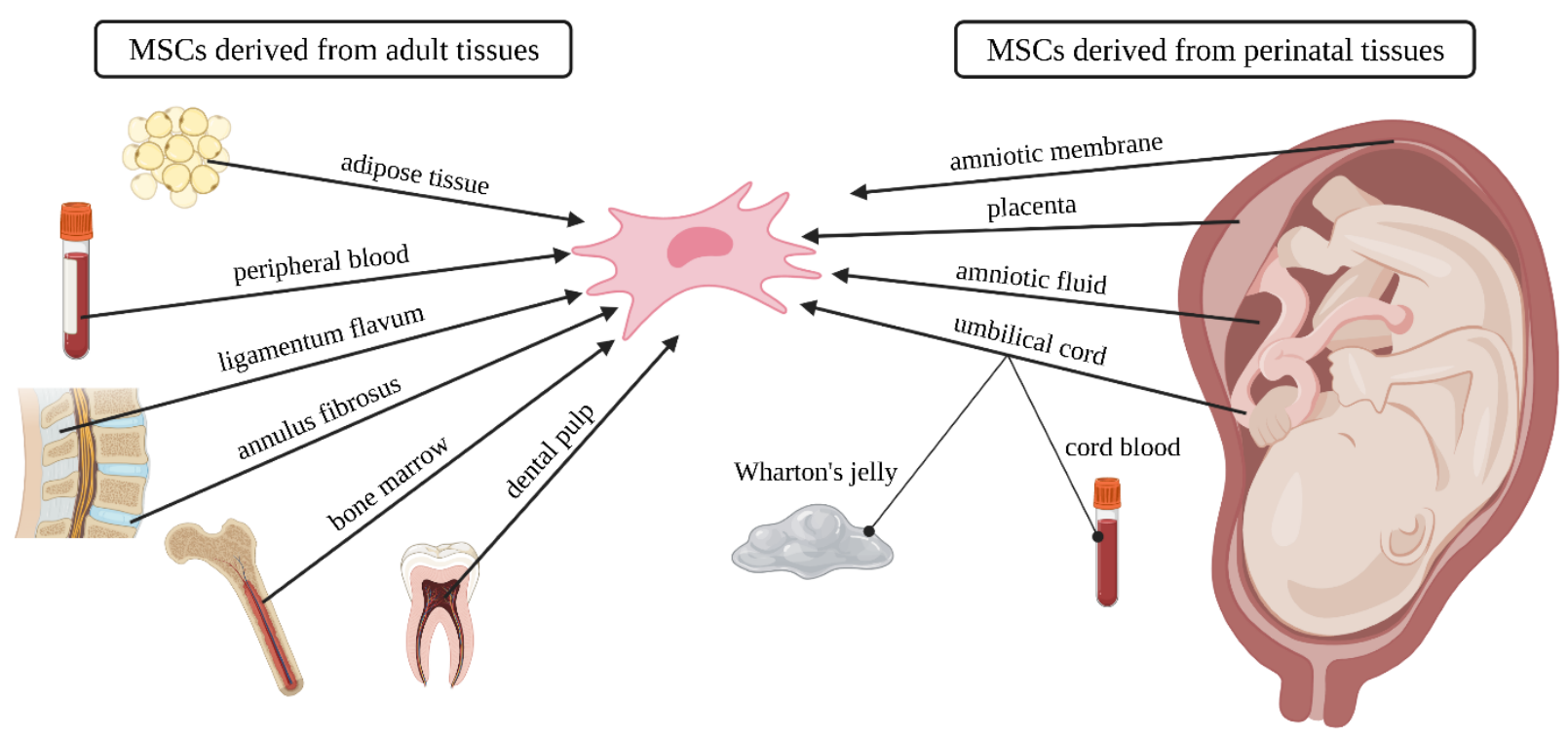

FIGURE 2 Mesenchymal stem cell sources can be split into adult tissues like adipose tissue, peripheral blood, ligamentum flavum, annulus fibrosus, bone marrow, or dental pulp, and perinatal tissues including amniotic membrane, amniotic fluid, placenta, umbilical cord Wharton's jelly and umbilical blood. Created with BioRender.com 
matic digestion, which is the most effective because it provides high level of cellular homogeneity and sufficient number of cells to start culture [51-53].

\section{Immunomodulatory properties of MSCs and their secretome}

MSCs exhibit multiple immunomodulatory properties such as immunotolerance, immunosuppression, apoptotic inhibition and the ability to migrate toward the site of inflammation [40]. Such a broad spectrum of activity is a highly desirable feature considering the complexity of the autoimmune mechanisms involved in SLE. MSCs act simultaneously via cell contact-dependent mechanisms and through their secretome, which consists of soluble molecules and EVs. MSCs' immunosuppressive activity is conditioned by their exposure to pro-inflammatory agents: IFN- $\gamma$, TNF, IL- $1 \beta$, and other microenvironmental factors, such as heat-shock proteins and RNAs. Depending on IL-10 signaling, Tr1 cells produce high levels of IL-10 and IFN- $\beta$. INF- $\beta$ induces toll-like receptor 3 through STAT1 signaling, resulting in a switch of MSCs into MSC1 immunophenotype, which exhibits enhanced immunosuppressive activity. As upregulation of these agents is noted in SLE, they might stimulate anti-inflammatory action of allogenic MSCs introduced in form of therapy. MSCs contribute to the inhibition of mDCs formation, as well as promotion of pDCs, leading to the development of Treg cells and the polarization of the macrophage phenotype to the immunosuppressive type M2 through the production of IL-10. As it was shown that numbers of pDCs and Tregs are downregulated in SLE, stimulation of their development might be beneficial for SLE treatment, as Treg cells are essential for suppression of immune response. Moreover, in the presence of inflammatory agents, such as IFN- $\gamma$, MSCs upregulate IDO expression, resulting in T cell apoptosis. Studies shown that in SLE MSCs present downregulation of IDO pathway. Therefore it is worth to place attention on the potential benefits of allogenic transplantation of MSCs, or even application of their paracrine factors. MSCs present capability to suppress B cells proliferation, chemotaxis, differentiation to plasma cells and autoantibody production. Additionally, these cells tend to inhibit NK cell cytotoxic activity, leading to an increase in IL-6 and STAT3 signaling, thus limiting neutrophil involvement and decreasing ROS production. Moreover, the action of MSCs reduces the level of IFN- $\boldsymbol{\gamma}[17,25,54,55]$. All of these pathways are affected in SLE, and MSCs carry potential to overcome vast amount of disturbances related to dysregulation of immune system in this disease.

B cells play a central role in SLE course, as they drive chronic inflammation by production of autoantibodies. Several studies were conducted regarding the effect of MSC stimulation on B cells. Cocul- tures of umbilical cord mesenchymal stem cells (UC-MSCs) and B cells from murine spleen resulted in suppression of their proliferation and differentiation. It was suggested that these mechanisms could be mediated by soluble factors produced by UCMSCs [56]. Furthermore, the effect of MSCs on B cell modulation was examined on cells isolated from C57BL/ 6 mice. In this study, BM-MSCs were expanded and B cells isolated from spleen were activated with lipopolysaccharide. Activated B cells were co-cultured with MSCs, resulting in suppression of B cell proliferation. Additionally, the impact of conditioned media from MSCs cultures was studied. In vitro stimulation caused suppression of B cells differentiation, while in vivo experiments resulted in inhibition of IgM and IgG1 secretion [57]. Additional studies were conducted on human MSCs derived from multiple tissues: umbilical cord matrix, bone marrow and adipose tissue. Studies revealed that cells from every tissue were able to prevent the activation of TCD4+, TCD8+ and NK cells. However, regarding their impact on $\mathrm{B}$ cells, inhibitory effect was provided only by cells isolated from bone marrow and adipose tissue, while MSCs from umbilical cord matrix had no effect on B cell suppression [58]. In light of such reports, it is important to highlight the need for deeper investigation regarding impact of MSCs on B cells activity.

Multiple components of MSCs secretome exhibit immunosuppressive properties, e.g. TGF- $\beta$ stimulate Treg activation and suppression of DCs maturation [55]. In turn, matrix metalloproteinases allow for cleavage of multiple molecules, including MSCs-derived CCL2, resulting in formation antagonistic form of CCL2 [59], which tends to suppress chemotaxis and activation of Th17. Other molecules secreted by MSCs include: IL-1Ra capable to inhibit B cell differentiation, and leukemia inhibitory factor, showing potential to suppress Th17 cell differentiation, as well as activate STAT3 phosphorylation and Treg proliferation. Furthermore, secretion of prostaglandin E2 allows to induce production of IL-10 by macrophages and plasmacytoid DCs, and to inhibit production of TNF by myeloid DCs. MSCs secretome also contains molecules such as: Gal-1 with the capacity to inhibit proliferation of TCD4+ and TCD8+ cells, and Gal-9, supporting Th17 and Th1 cell death. Moreover, PGE2 and IDO tend to inhibit the proliferation and cytotoxicity of resting NK cells $[11,55]$. Through the inhibition of pro-inflammatory elements of immune system: Th17 cells, NK cells, B cells, TNF and simultaneous stimulation of immunomodulators: Treg cells, IL-10, PGE2, IDO, MSCs secretome presents the ability to regulate chronic inflammation in SLE. Although MSCs have a therapeutic effect in the treatment of autoimmune diseases, it should be remembered that precise mechanism of their action is not fully understood. Therefore, to provide a better overview of these in- 
teractions, and to increase the efficacy and safety of this therapeutic approach, it is essential to conduct more studies [60].

\section{Extracellular vesicles derived from MSCs}

Extracellular vesicles are important immunomodulators excreted by MSCs, forming the basis of the mechanism ensuring intercellular communication without cell-to-cell contact $[61,62]$. The most effective immunosuppressive action of MSCs is provided by the type of EVs known as exosomes [10], with a diameter between $30-100 \mathrm{~nm}$. Biogenesis of exosomes starts by generation of early endosome. Then membrane of endosome undergo invagination, forming intraluminal vesicles, which may carry: proteins, mRNA, miRNA, long noncoding RNA, lipids, ceramide, sphingomyelin and other molecules derived from cytosol of the mother cell. Late endosome is transformed into multivesicular body, which is able to fuse with plasma membrane, leading to release of exosomes to the extracellular environment (Fig. 3) [63,64]. Exosomes are surrounded by double phospholipid membrane, and carry the set of surface molecules inherited from the source cell. Characterization of those vesicles is possible by detection of markers such as: Alix, TSG101, Hsp60, Hsp70, Hsp90 and particular tetraspins. Those subcellular particles present ability to fuse with the surface membrane of targeted cell and are able to induce precise functional changes $[61,65]$.

\section{Treatment of SLE using MSCs and their secretome}

It is important to develop alternative therapies using MSCs and their secretome because there is no specific cure for SLE. Immunomodulation of MSCs is exerted not only via cell-to-cell contact, but also in a paracrine manner. There are several animal studies and human trials on the use of these properties in the treatment of SLE. Despite the fact that the secretome of MSCs have not yet been tested in SLE treatment, it appears to be beneficial in therapy of other diseases.

Application of murine models in research on SLE therapy development is indispensable, and provides an excellent opportunity to evaluate its efficiency, dosage and toxicity. In addition, mouse

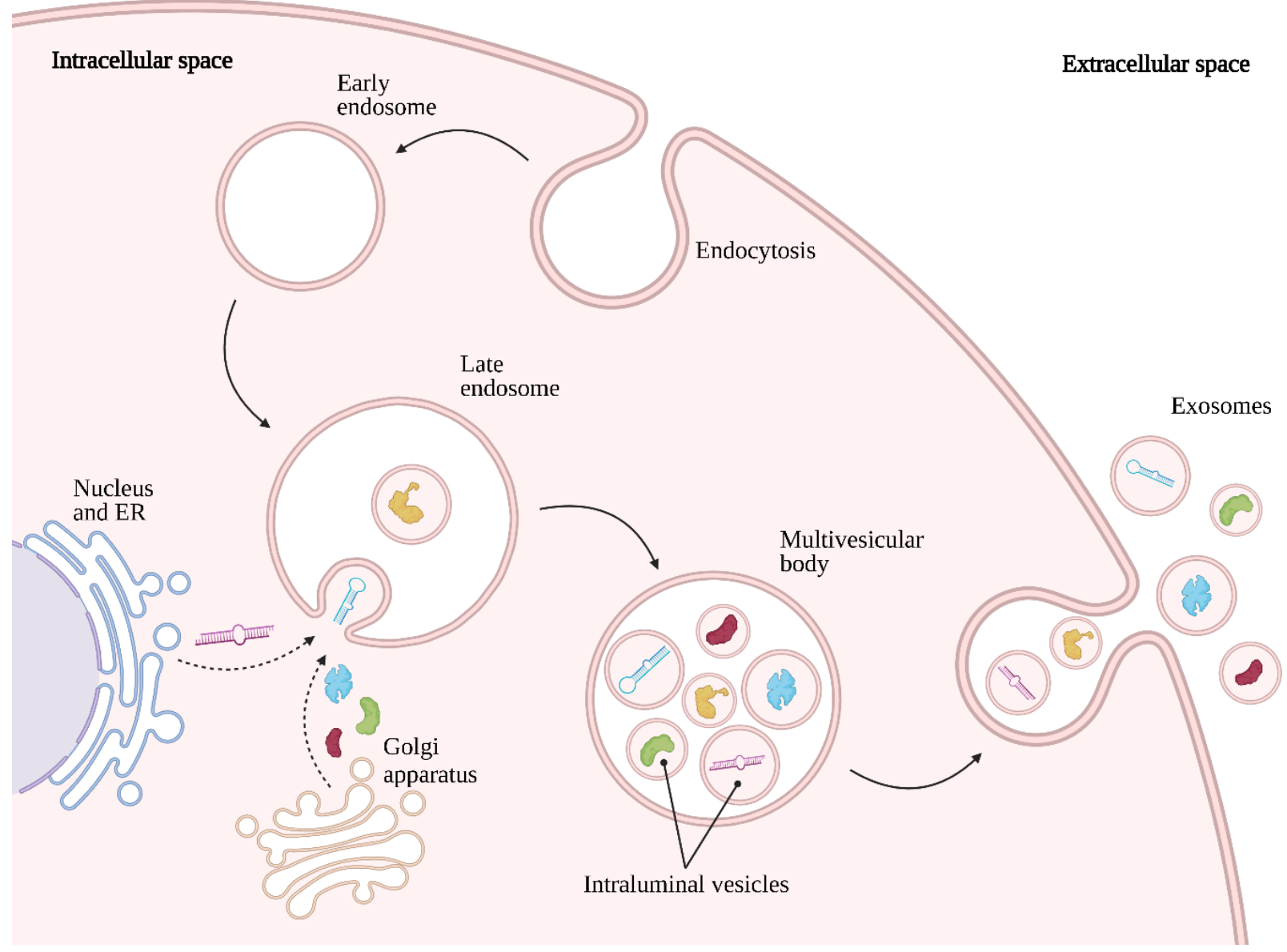

FIGURE 3 Schematic representation of exosome biogenesis. Process of exosome formation begins with endocytosis, leading to formation of early endosome. Then molecules such as nucleic acids, proteins and lipids, of both intra and extracellular origin, invaginate into the lumen of late endosome, giving rise to intraluminal vesicles. Late endosome transform into multivesicular body, which is capable to undergo fusion with cell membrane and release of exosomes to the extracellular space. Created with BioRender.com 
models provide controlled experimental conditions rarely achievable in human studies. SLE studies on mouse models are conducted in a germ-free environment, unlike clinical trials, since humans are exposed to pathogens that activate immune response mechanisms. In addition, murine models allow carry research on non-treated cohort, when it is not possible is relation to clinical studies, as drugs are administered to SLE patients even during the period of disease remission. Hence, mouse models provide the possibility of conducting complex studies under controlled conditions and on untreated groups. Although, it is important to keep in mind that there are some differences between mouse and human immune system. Studies on mouse models allow to collect statistically complete data that may be translatable to the human condition [66].

A characteristic feature of SLE is variability in the manifestation of symptoms, as well as in their severity. Each mouse model provides a unique set of SLE features. However, none of them provide the entire spectrum, but only limited aspects of SLE. Most SLE studies are performed in spontaneous mouse models such as New Zealand Black/White F1 (NZB/W F1), Murphy Roths Large/lymphoproliferative (MRL/lpr), New Zealand Mixed strains: NZM2410, NZM 2328 or BXSB/Yaa [67]. However, application of induced models, such as pristan-induced, enables to create specific conditions, allowing for dissection of mechanisms that may trigger SLE development, especially in context of genetic factors contribution $[67,68]$.

\section{MSCs transplantation for SLE treatment - preclinical and clinical studies}

The use of stem cells or stem cell products is considered as a highly promising method of effective treatment, especially for diseases such as SLE, when the physiological processes of tissue regeneration are inhibited by underlying pathological conditions [52]. Preclinical and clinical trials were already conducted regarding the application of MSCs in SLE treatment. The results indicate that curation with MSCs is effective and can lead to long-term improvement in health. Its safety was also analyzed, and it was concluded that this type of therapy is associated with a low risk of side effects [45,69-78]. It should be noted, however, that in the case of insufficient compliance of a large histocompatibility complex, graft rejection may lead to a significant deterioration of the patient's health [79]. MSCs derived from perinatal tissues have the greatest potential for use in SLE treatment, as they present remarkably low immunogenicity. Additionally, hWJMSCs express low levels of MHC class I and more importantly, do not exhibit expression of MHC class II [80] and co-stimulatory molecules such as CD40, CD40L, CD80 or CD86. In vitro studies show that
MHC-mismatched MSCs do not trigger proliferative T-cell response in the allogeneic mixed lymphocyte reaction. Thus, hWJ-MSCs are immune-privileged cells which cannot activate alloreactive T cells, and are able to escape immune recognition and clearance. Therefore, allogenic transplantation of hWJMSCs presents high potential for effective and safe method of SLE treatment [38].

The results of studies in murine SLE models also provide evidence that allogeneic MSCs transplantation resulted in SLE remission and reduction of organ failure [77]. There was also noted a decrease of anti-dsDNA antibodies, C3 complement, Th1 and Th17 lymphocytes, TNF- $\alpha$, IL-6, IL-17 and B cells precursors, when the number of Treg cells and levels of IL-4 and IL-10 raised [31]. In trials on lupus nephritis mouse model, MSCs transplantation resulted in decrease of Th1 cytokines: IFN- $\gamma$, IL-2, TNF- $\alpha$, IL-6, IL-12, and caused increase of Th2 cytokines: IL-4, IL-10 [75].

In human studies the effect of UC-MSCs administration on the regulation of Treg and Th17 cells was investigated in SLE patients. One week after infusion, a decrease in TNF- $\alpha$ was noted. Moreover, increased expression of Treg and Foxp3 was observed from one week to 3 months after transplantation. Increase of TGF- $\beta$ level was present in period from week one to 12 months after procedure, while the authors reported downregulation of IL-17 and decreased number of Th17 in term from 3 to 12 months after treatment. In vitro tests co-culturing UC-MSCs with patient-derived peripheral blood mononuclear cells, showed that TGF- $\beta$, IL-6, PGE2, and IDO levels increased. Therefore, it was concluded that UC-MSCs acted by regulating TGF- $\beta$ and PGE. Moreover, it was hypothesized that SLE microenvironment stimulates the action of UCMSCs, since both types of cells in separate cultures, exhibited significantly less production of TGF- $\beta$, IL-6, PGE2 and IDO [81]. Other clinical trials have assessed the long-term safety of UC-MSCs allograft in patients with SLE. Adverse effects and health parameters were monitored before, immediately after infusion and 1, 2, 4 and 6 years after the procedure. The studies showed that the only side effects were a feeling of warmth and dizziness for 5 minutes after the injection, seen in only one out of nine patients. Hence, studies have shown that UC-MSCs treatment was safe [69].

Overall, studies demonstrate that treatment with MSCs result in the autoinflammation regression, improvement of organ and tissue function, and led to SLE remission [66,69,70,75,76,81]. However, some studies suggested that only small fraction of MSCs was able to undergo engraftment in host tissues, suggesting that immunosuppressive influence of MSCs can undergo reduction by poor ex vivo expansion of cells [12]. 


\section{MSCs secretome - attractive candidate for SLE treatment}

Numerous studies have shown that paracrine mechanisms, especially those triggered by the action of EV, have crucial importance for the immunomodulatory and regenerative properties of MSCs. Additionally, MSCs are noted as one of the most effective producers of EVs $[65,82]$. Therapeutic application of MSCs secretome components is more advantageous than the use of MSCs, as they are not subjected to the strict regulations which are typical for stem cell therapy. Moreover, their intravenous administration appears to be safe for clinical use. EVs are characterized by efficient, simple method of production and isolation. Consequently, standardization of its production on an industrial scale appears to be easier to organize compared to MSCs. Additionally, EVs are stable and can easily be stored for long-term usage by simple freezing $[11,12]$. To ensure reproducibility and minimize the risk of discrepancy, the International Society for Extracellular Vesicles has published guidelines for the most appropriate EVs separation methods, as well as recommendations for their appropriate characterization [83].

Recently, a number of pre-clinical and clinical studies regarding the use of MSCs secretome have been carried out. For spinal cord injury in rats, treatment by administering hWJ-MSC and their conditioned media (CM) containing extracellular vesicles was compared. In both cases, there was noted increase in the amount of white and gray matter and upregulation of axonal growth-associated genes expression, resulting in significant improvement in health. Moreover, application of CM resulted in upregulation of axonal sprouting and reduction in the astrocyte response in the site of administration [84]. Studies in rodent models of immune disorders, including multiple sclerosis [85] and Alzheimer's disease [47], have confirmed the anti-inflammatory properties of the MSCs secretome [84]. In other studies on an acute colitis mouse model, a filtrate of MSC-conditioned medium was administrated as a treatment agent to the mice. Subsequently, the treated mice exhibited a reduction of inflammation, upregulation of Treg cells and TGF- $\beta$ levels together with downregulation of IL-10 and IL-17 levels. Therefore, it has been suggested that MSCs secretome exhibit immunomodulatory properties and demonstrate potential for significant reduction of inflammation [86].

Only a few clinical trials involving the components of MSCs secretome have been reported. One study was focused on alveolar bone atrophy treatment with CM application. Scaffold grafts soaked with CM of bone marrow MSCs were implanted to patients revealing a downregulation of cells involved in the induction of inflammation [87].

In other clinical trials, the therapeutic effect of injecting components selected from MSCs secre- tome in pattern hair loss [46] and alopecia [88]. Both experiments resulted in hair densification. Trials which examine therapeutic effects of the MSCs secretome lead to effective long-term improvement in health and no side effects.

Since the start of the COVID-19 pandemic in December 2019 [89], in ClinicalTrials.gov database have been registered 8 clinical trials on the application of the MSCs secretome to treat symptoms caused by severe acute respiratory syndrome coronavirus 2 (SARS-CoV-2). This may be considered as a beneficial fact for the development and popularization of therapy with the use of the MSCs secretome, as it may boost the number of scientific reports related to its application as a treatment agent. However, despite the growing number of clinical trials using the MSCs secretome as a therapeutic agent, such an approach is still missing in research on treatment strategy for SLE.

\section{Conclusions}

The unique immunomodulatory properties of MSCs, especially of those derived from perinatal tissues, offer opportunity to develop a highly effective treatment strategy for SLE. Such therapy could be much more effective in maintaining remission of SLE and be associated with a lower risk of side effects than the common drug-based approach. Numerous studies have suggested that application of MSCs as a therapeutic agent is effective and safe method for SLE treatment.

Nevertheless, the therapeutic effect of stem cellbased therapy may be limited, depending on the destination site, as their ability to penetrate tissue varies in relation to the barriers the MSCs must migrate. Studies provide evidences that only a fraction of transplanted cells successfully undergo engraftment and therefore the immunosuppressive effect of such therapy might be significantly reduced. Furthermore, there are legitimate concerns about the reproducibility of the effects of MSC-based therapies. In addition, while MSCs transplantation is considered to be safe, several important issues have to be taken into account regarding their clinical application such as a chance to undergo senescence, loss of functionality, oncogenic progression or possibility of an HLA mismatch occurrence.

The available data suggests that treatment with periodic intravenous administration of immunomodulatory agents derived from hWJ-MSCs conditioned medium is more advantageous than therapy with intravenous administration of hWJ-MSCs. The application of isolated paracrine factors eliminates the risks related to stem cell- based therapy. Moreover, components of MSCs secretome present invaluable feature - their ability to reach the affected site is much higher comparing to stem cell-based treatment strategy. Moreover, as MSCs are efficient producers of paracrine compounds, including EVs, 
and the method of EVs production and isolation appears to be scalable, the cost of therapy based on the use of MSCs secretome components is expected to be affordable.

With regard to the therapies applying regenerative medicine approach, regular administration of MSCs and components of their secretome appears to be the most promising concept for SLE treatment. The effectiveness of this strategy should be carefully examined. Modifications in the proportions of these agents, as well as their simultaneous or interchangeable application should also be considered.

\section{Ethical approval}

The conducted research is not related to either human or animal use.

\section{Acknowledgements \\ Not applicable.}

\section{Corresponding author}

Marta Dyszkiewicz-Konwińska, Department of Biomaterials and Experimental Dentistry, 70 Bukowska St., 60-812 Poznań, Poland and Department of Anatomy, Poznan University of Medical Sciences, 6 Święcickiego St., 60-781 Poznań, Poland, Tel./Fax: +48 61 8546565, e-mail: m.dyszkiewicz@ump.edu.pl.

\section{Conflict of interests statement}

The authors declare they have no conflict of interest.

\section{References}

1. Kapsogeorgou EK., Tzioufas AG. Autoantibodies in Autoimmune Diseases: Clinical and Critical Evaluation. Isr Med Assoc J. 2016;18:519-524.

2. Lerner A, Jeremias P, Matthias T. The World Incidence and Prevalence of Autoimmune Diseases is Increasing. Int J Celiac Dis. 2016;3(4); DOI:10.12691/ijcd-3-4-8.

3. Cooper GS, Bynum MLK, Somers EC. Recent insights in the epidemiology of autoimmune diseases: Improved prevalence estimates and understanding of clustering of diseases. J Autoimmun. 2009;33(3-4) DOI:10.1016/j.jaut.2009.09.008.

4. Singh RR, Yen EY. SLE mortality remains disproportionately high, despite improvements over the last decade. Lupus. 2018;27(10) DOI:10.1177/0961203318786436.

5. Stojan G, Petri M. Epidemiology of systemic lupus erythematosus: an update. Curr Opin Rheumatol. 2018;30(2); DOI:10.1097/ BOR.0000000000000480.

6. Rees F, Doherty M, Grainge MJ, Lanyon P, Zhang W. The worldwide incidence and prevalence of systemic lupus erythematosus: a systematic review of epidemiological studies. Rheumatology. 2017;56(11); DOI:10.1093/rheumatology/kex260.

7. Montero-López E, Santos-Ruiz A, Navarrete-Navarrete N, Ortego-Centeno N, Pérez-García M, Peralta-Ramírez MI. The effects of corticosteroids on cognitive flexibility and decision-making in women with lupus. Lupus. 2016;25(13); DOI:10.1177/0961203316642313.

8. Christopeit M, Schendel M, Föll J, Müller LP, Keysser G, Behre G. Marked improvement of severe progressive systemic sclerosis after transplantation of mesenchymal stem cells from an allogeneic haploidentical-related donor mediated by ligation of CD137L. Leukemia. 2008;22(5); DOI:10.1038/sj.leu.2404996.

9. Ezquer FE, Ezquer ME, Parrau DB, Carpio D, Yañez AJ, Conget PA. Systemic Administration of Multipotent Mesenchymal Stromal Cells Reverts Hyperglycemia and Prevents Nephropathy in Type 1 Diabetic Mice. Biol Blood Marrow Transplant. 2008;14(6); DOI:10.1016/j. bbmt.2008.01.006.

10. Cosenza S, Toupet K, Maumus M, Luz-Crawford P, Blanc-Brude O, Jorgensen C, Noël D. Mesenchymal stem cells-derived exosomes are more immunosuppressive than microparticles in inflammatory arthritis. Theranostics. 2018;8(5); DOI:10.7150/thno.21072.

11. Eleuteri S, Fierabracci A. Insights into the Secretome of Mesenchymal Stem Cells and Its Potential Applications. Int J Mol Sci. 2019;20(18); DOI:10.3390/ijms20184597.
12. Sharma J, Hampton JM, Valiente GR, Wada T, Steigelman H, Young MC, Spurbeck RR, Blazek AD, Bösh S, Jarjour WN, Young NA. Therapeutic Development of Mesenchymal Stem Cells or Their Extracellular Vesicles to Inhibit Autoimmune-Mediated Inflammatory Processes in Systemic Lupus Erythematosus. Front Immunol. 2017;8; DOI:10.3389/ fimmu.2017.00526.

13. GfK Roper. Lupus Awareness Survey for the Lupus Foundation of America. GfK Roper Public Aff Corp Commun. 2012

14. MichetI CJ, McKenna CH, Elveback LR, Kaslov RA, Kurland LT. Epidemiology of Systemic Lupus Erythematosus and Other Connective Tissue Diseases in Rochester, Minnesota, 1950 Through 1979. Mayo Clin Proc. 1985;60(2); DOI:10.1016/S0025-6196(12)60294-8.

15. Flower C, Hennis AJM, Hambleton IR, Nicholson GD, Liang MH. Systemic lupus erythematosus in an Afro-Caribbean population: Incidence, clinical manifestations and survival in the Barbados national lupus registry. Arthritis Care Res (Hoboken). 2012; DOI:10.1002/acr.21656.

16. Zen M, Ghirardello A, Iaccarino L, Tonon M, Campana C, Arienti S, Rampudda M, Canova M, Doria A. Hormones, immune response, and pregnancy in healthy women and SLE patients. Swiss Med Wkly. 2010;140:187-201.

17. Azevedo PC, Murphy G, Isenberg DA. Pathology of Systemic Lupus Erythematosus: The Challenges Ahead. In: ; 2014. DOI:10.1007/978-1-4939-0326-9_1.

18. Ruperto N, Hanrahan L, Alarcón G, et al. International consensus for a definition of disease flare in lupus. Lupus. 2011;20(5); DOI:10.1177/0961203310388445.

19. Fernandez D, Kirou KA. What Causes Lupus Flares? Curr Rheumatol Rep. 2016;18(3); DOI:10.1007/s11926-016-0562-3.

20. Chen L, Morris DL, Vyse TJ. Genetic advances in systemic lupus erythematosus: an update. Curr Opin Rheumatol. 2017;29(5); DOI:10.1097/ BOR.0000000000000411.

21. Sullivan KE. Genetics of systemic lupus erythematosus. Rheum Dis Clin North Am. 2000;26(2); DOI:10.1016/S0889-857X(05)70137-X.

22. James JA, Robertson JM. Lupus and Epstein-Barr. Curr Opin Rheumatol. 2012;24(4); DOI:10.1097/BOR.0b013e3283535801.

23. Munoz LE, Gaipl US, Franz S, Sheriff A, Voll RE, Kalden JR, Herrmann M. SLE-a disease of clearance deficiency? Rheumatology. 2005;44(9); DOI:10.1093/rheumatology/keh693.

24. Jung J-Y, Suh C-H. Incomplete clearance of apoptotic cells in systemic lupus erythematosus: pathogenic role and potential biomarker. Int J Rheum Dis. 2015;18(3); DOI:10.1111/1756-185X.12568.

25. Zharkova O, Celhar T, Cravens PD, Satterthwaite AB, Fairhurst A-M, Davis LS. Pathways leading to an immunological disease: systemic lupus erythematosus. Rheumatology. 2017;56(suppl_1); DOI:10.1093/ rheumatology/kew427.

26. Morel L. Immunometabolism in systemic lupus erythematosus. Nat Rev Rheumatol. 2017;13(5); DOI:10.1038/nrrheum.2017.43.

27. Le Blanc K, Ringdén O. Immunomodulation by mesenchymal stem cells and clinical experience. J Intern Med. 2007;262(5); DOI:10.1111/j.1365-2796.2007.01844.x.

28. Tyndall A, Gratwohl A. Haemopoietic stem and progenitor cells in the treatment of severe autoimmune diseases. Ann Rheum Dis. 1996;55(3); DOI:10.1136/ard.55.3.149.

29. Zhu Y, Feng X. Genetic contribution to mesenchymal stem cell dysfunction in systemic lupus erythematosus. Stem Cell Res Ther. 2018;9(1); DOI:10.1186/s13287-018-0898-x.

30. Sun L, Zhang H, Feng X, Hou Y, Lu L, Fan L. Abnormality of bone marrow-derived mesenchymal stem cells in patients with systemic lupus erythematosus. Lupus. 2007;16(2); DOI:10.1177/0961203306075793.

31. Fathollahi A, Gabalou NB, Aslani S. Mesenchymal stem cell transplantation in systemic lupus erythematous, a mesenchymal stem cell disorder. Lupus. 2018;27(7); DOI:10.1177/0961203318768889.

32. Wang D, Sun L. Systemic Lupus Erythematosus. In: Chen X. D., ed. $A$ Roadmap to Non-Hematopoietic Stem Cell-Based Therapeutics. Academic Press.; 2019:143-172.

33. Tang Y, Ma X, Zhang H, Gu Z, Hou Y, Gilkeson GS, Lu L, Zeng X, Sun L. Gene Expression Profile Reveals Abnormalities of Multiple Signaling Pathways in Mesenchymal Stem Cell Derived from Patients with Systemic Lupus Erythematosus. Clin Dev Immunol. 2012;2012; DOI:10.1155/2012/826182.

34. Xu J. Therapeutic Applications of Mesenchymal Stem Cells for Systemic Lupus Erythematosus. In: ; 2018. DOI:10.1007/5584_2018_212.

35. Carrion F, Nova E, Ruiz C, Diaz F, Inostroza C, Rojo D, Mönckeberg G, Figueroa F. Autologous mesenchymal stem cell treatment increased $\mathrm{T}$ regulatory cells with no effect on disease activity in two systemic lupus erythematosus patients. Lupus. 2010;19(3); DOI:10.1177/0961203309348983. 
36. Barbado J, Tabera S, Sánchez A, García-Sancho J. Therapeutic potential of allogeneic mesenchymal stromal cells transplantation for lupus nephritis. Lupus. 2018;27(13); DOI:10.1177/0961203318804922.

37. Wallace DJ. The evolution of drug discovery in systemic lupus erythematosus. Nat Rev Rheumatol. 2015;11(10); DOI:10.1038/ nrrheum.2015.86.

38. Liang J, Sun L. Mesenchymal stem cells transplantation for systemic lupus erythematosus. Int J Rheum Dis. 2015;18(2); DOI:10.1111/1756-185X.12531.

39. Fors Nieves CE, Izmirly PM. Mortality in Systemic Lupus Erythematosus: an Updated Review. Curr Rheumatol Rep. 2016;18(4); DOI:10.1007/ s11926-016-0571-2.

40. Joerger-Messerli MS, Marx C, Oppliger B, Mueller M, Surbek D V., Schoeberlein A. Mesenchymal Stem Cells from Wharton's Jelly and Amniotic Fluid. Best Pract Res Clin Obstet Gynaecol. 2016;31; DOI:10.1016/j. bpobgyn.2015.07.006.

41. Araújo AB, Salton GD, Furlan JM, Schneider N, Angeli MH, Laureano ÁM, Silla L, Passos EP, Paz AH. Comparison of human mesenchymal stroma cells from four neonatal tissues: Amniotic membrane, chorionic membrane, placental decidua and umbilical cord. Cytotherapy. 2017;19(5); DOI:10.1016/j.jcyt.2017.03.001.

42. Dominici M, Le Blanc K, Mueller I, Slaper-Cortenbach I, Marini F., Krause DS, Deans RJ, Keating A, Prockop DJ, Horwitz EM. Minimal criteria for defining multipotent mesenchymal stromal cells. The International Society for Cellular Therapy position statement. Cytotherapy. 2006;8(4); DOI:10.1080/14653240600855905.

43. Friedenstein AJ., Gorskaja JF., Kulagina NN. Fibroblast precursors in normal and irradiated mouse hematopoietic organs. Exp Hematol 1976;4:267-274;

44. Calloni R, Cordero EAA, Henriques JAP, Bonatto D. Reviewing and Updating the Major Molecular Markers for Stem Cells. Stem Cells Dev. 2013;22(9); DOI:10.1089/scd.2012.0637.

45. Choi EW, Lee HW, Shin IS, Park JH, Yun TW, Youn HY, Kim S-J. Comparative Efficacies of Long-Term Serial Transplantation of Syngeneic, Allogeneic Xenogeneic, or CTLA4Ig-Overproducing Xenogeneic Adipose Tissue-Derived Mesenchymal Stem Cells on Murine Systemic Lupus Erythematosus. Cell Transplant. 2016;25(6); DOI:10.3727/096368915X689442.

46. Shin H, Ryu HH, Kwon O, Park B-S, Jo SJ. Clinical use of conditioned media of adipose tissue-derived stem cells in female pattern hair loss: a retrospective case series study. Int J Dermatol. 2015;54(6); D0I:10.1111/ ijd.12650.

47. Mita T, Furukawa-Hibi Y, Takeuchi H, Hattori H, Yamada K, Hibi H, Ueda $\mathrm{M}$, Yamamoto A. Conditioned medium from the stem cells of human dental pulp improves cognitive function in a mouse model of Alzheimer's disease. Behav Brain Res. 2015;293; DOI:10.1016/j.bbr.2015.07.043.

48. Tracy SA, Ahmed A, Tigges JC, Ericsson M, Pal AK, Zurakowski D, Fauza DO. A comparison of clinically relevant sources of mesenchymal stem cell-derived exosomes: Bone marrow and amniotic fluid. J Pediatr Surg. 2019;54(1); DOI:10.1016/j.jpedsurg.2018.10.020.

49. Harris L, Vangsness CT. Mesenchymal Stem Cell Levels of Human Spinal Tissues. Spine (Phila Pa 1976). 2018;43(9); DOI:10.1097/ BRS.0000000000002401.

50. Davies JE, Walker JT, Keating A. Concise Review: Wharton's Jelly: The Rich, but Enigmatic, Source of Mesenchymal Stromal Cells. Stem Cells Transl Med. 2017;6(7); DOI:10.1002/sctm.16-0492.

51. Ding D-C, Chang Y-H, Shyu W-C, Lin S-Z. Human Umbilical Cord Mesenchymal Stem Cells: A New Era for Stem Cell Therapy. Cell Transplant. 2015;24(3); DOI:10.3727/096368915X686841.

52. La Rocca G. Novel Immunomodulatory Markers Expressed by Human WJ-MSC: an Updated Review in Regenerative and Reparative Medicine. Open Tissue Eng Regen Med J. 2012;5(1); DOI:10.2174/18750435012 05010050.

53. Tong CK, Vellasamy S, Chong Tan B, Abdullah M, Vidyadaran S, Fong Seow H, Ramasamy R. Generation of mesenchymal stem cell from human umbilical cord tissue using a combination enzymatic and mechanical disassociation method. Cell Biol Int. 2011;35(3); DOI:10.1042/CBI20100326.

54. Rubtsov Y, Goryunov K, Romanov A, Suzdaltseva Y, Sharonov G, Tkachuk V. Molecular Mechanisms of Immunomodulation Properties of Mesenchymal Stromal Cells: A New Insight into the Role of ICAM-1. Stem Cells Int. 2017;2017; DOI:10.1155/2017/6516854.

55. Pistoia V, Raffaghello L. Mesenchymal stromal cells and autoimmunity. Int Immunol. 2017;29(2); DOI:10.1093/intimm/dxx008.

56. Che N, Li X, Zhou S, Liu R, Shi D, Lu L, Sun L. Umbilical cord mesenchymal stem cells suppress B-cell proliferation and differentiation. Cell Immunol. 2012;274(1-2); DOI:10.1016/j.cellimm.2012.02.004.

57. Asari S, Itakura S, Ferreri K, Liu C-P, Kuroda Y, Kandeel F, Mullen Y. Mesenchymal stem cells suppress B-cell terminal differentiation. Exp Hematol. 2009;37(5); DOI:10.1016/j.exphem.2009.01.005.
58. Ribeiro A, Laranjeira P, Mendes S, Velada I, Leite C, Andrade P, Santos F, Henriques A, Grãos M, Cardoso CMP, Martinho A, Pais Ml, da Silva C, Cabral J, Trindade H, Paiva A. Mesenchymal stem cells from umbilical cord matrix, adipose tissue and bone marrow exhibit different capability to suppress peripheral blood B, natural killer and T cells. Stem Cell Res Ther. 2013;4(5); DOI:10.1186/scrt336.

59. Rafei M, Campeau PM, Aguilar-Mahecha A, Buchanan M, Williams P, Birman E, Yuan S, Young YK, Boivin M-N, Forner K, Basik M, Galipeau J. Mesenchymal Stromal Cells Ameliorate Experimental Autoimmune Encephalomyelitis by Inhibiting CD4 Th17 T Cells in a CC Chemokine Ligand 2-Dependent Manner. J Immunol. 2009;182(10); DOI:10.4049/ jimmunol.0803962.

60. Cheng R-J, Xiong A-J, Li Y-H, Pan S-Y, Zhang Q-P, Zhao Y, Liu Y, Marion TN. Mesenchymal Stem Cells: Allogeneic MSC May Be Immunosuppressive but Autologous MSC Are Dysfunctional in Lupus Patients. Front Cell Dev Biol. 2019;7; DOI:10.3389/fcell.2019.00285.

61. Perez-Hernandez J, Redon J, Cortes R. Extracellular Vesicles as Therapeutic Agents in Systemic Lupus Erythematosus. Int J Mol Sci. 2017;18(4); DOI:10.3390/ijms18040717.

62. Luan X, Sansanaphongpricha K, Myers I, Chen H, Yuan H, Sun D. Engineering exosomes as refined biological nanoplatforms for drug delivery. Acta Pharmacol Sin. 2017;38(6); DOI:10.1038/aps.2017.12.

63. Raposo G, Stoorvogel W. Extracellular vesicles: Exosomes, microvesicles, and friends. J Cell Biol. 2013;200(4); DOI:10.1083/jcb.201211138.

64. Mathivanan S, Simpson RJ. ExoCarta: A compendium of exosomal proteins and RNA. Proteomics. 2009;9(21); DOI:10.1002/pmic.200900351.

65. Qiu G, Zheng G, Ge M, Wang J, Huang R, Shu Q, Xu J. Mesenchymal stem cell-derived extracellular vesicles affect disease outcomes via transfer of microRNAs. Stem Cell Res Ther. 2018;9(1); DOI:10.1186/ s13287-018-1069-9.

66. Celhar T, Fairhurst A-M. Modelling clinical systemic lupus erythematosus: similarities, differences and success stories. Rheumatology. December 2016; DOI:10.1093/rheumatology/kew400.

67. Li W, Titov AA, Morel L. An update on lupus animal models. Curr Opin Rheumatol. 2017;29(5); DOI:10.1097/BOR.0000000000000412.

68. Xu Y, Zeumer L, Reeves WH, Morel L. Induced Murine Models of Systemic Lupus Erythematosus. In: ; 2014. DOI:10.1007/978-1-4939-0326-9_9.

69. Wang D, Niu L, Feng X, Yuan X, Zhao S, Zhang H, Liang J, Zhao C, Wang H, Hua B, Sun L. Long-term safety of umbilical cord mesenchymal stem cells transplantation for systemic lupus erythematosus: a 6-year follow-up study. Clin Exp Med. 2017;17(3); DOI:10.1007/s10238-016-0427-0.

70. Zhang Y, Xia Y, Ni S, Gu Z, Liu H. Transplantation of umbilical cord mesenchymal stem cells alleviates pneumonitis of MRL/lpr mice. J Thorac Dis. 2014;6:109-117;

71. Li X, Wang D, Liang J, Zhang H, Sun L. Mesenchymal SCT ameliorates refractory cytopenia in patients with systemic lupus erythematosus. Bone Marrow Transplant. 2013;48(4); DOI:10.1038/bmt.2012.184.

72. Jang E, Jeong M, Kim S, Jang K, Kang B-K, Lee DY, Bae S-C, Kim KS, Youn J. Infusion of Human Bone Marrow-Derived Mesenchymal Stem Cells Alleviates Autoimmune Nephritis in a Lupus Model by Suppressing Follicular Helper T-Cell Development. Cell Transplant. 2016;25(1); DOI:10.3727/096368915X688173.

73. Thiel A, Yavanian G, Nastke M-D, Morales P, Kouris NA, Kimbrel EA, Lanza R. Human embryonic stem cell-derived mesenchymal cells preserve kidney function and extend lifespan in NZB/W F1 mouse model of lupus nephritis. Sci Rep. 2016;5(1); DOI:10.1038/srep17685.

74. Mai S, Zou L, Tian X, Liao X, Luan Y, Han X, Wei Y, Wu Y, Kuang S, Yang Y, Ma J, Chen Q Yang J. Double-Edged Effect of Hydroxychloroquine on Human Umbilical Cord-Derived Mesenchymal Stem Cells Treating Lupus Nephritis in MRL/lpr Mice. Mol Pharm. 2018;15(5); DOI:10.1021/acs. molpharmaceut.7b01146.

75. Chang J-W, Hung S-P, Wu H-H, Wu W-M, Yang A-H, Tsai H-L, Yang L-Y Lee OK. Therapeutic Effects of Umbilical Cord Blood-Derived Mesenchymal Stem Cell Transplantation in Experimental Lupus Nephritis. Cell Transplant. 2011;20(2); DOI:10.3727/096368910X520056.

76. Zhang Z, Feng R, Niu L, Huang S, Deng W, Shi B, Yao G, Chen W, Tang X, Gao X, Feng X, Sun L. Human Umbilical Cord Mesenchymal Stem Cells Inhibit T Follicular Helper Cell Expansion through the Activation of iNOS in Lupus-Prone B6.MRL- Fas ${ }^{\text {lpr }}$ Mice. Cell Transplant. 2017;26(6); DOI:10.3727/096368917X694660.

77. Gu F, Molano I, Ruiz P, Sun L, Gilkeson GS. Differential effect of allogeneic versus syngeneic mesenchymal stem cell transplantation in MRL/lpr and (NZB/NZW)F1 mice. Clin Immunol. 2012;145(2); DOI:10.1016/j. clim.2012.08.012.

78. Che N, Li X, Zhang L, Liu R, Chen H, Gao X, Shi S, Chen W, Sun L. Impaired B Cell Inhibition by Lupus Bone Marrow Mesenchymal Stem Cells Is Caused by Reduced CCL2 Expression. J Immunol. 2014;193(10); DOI:10.4049/jimmunol.1400036. 
79. Li T, Xia M, Gao Y, Chen Y, Xu Y. Human umbilical cord mesenchymal stem cells: an overview of their potential in cell-based therapy. Expert Opin Biol Ther. 2015;15(9); DOI:10.1517/14712598.2015.1051528.

80. Zhou C, Yang B, Tian Y, Jiao H, Zheng W, Wang J, Guan F. Immunomodulatory effect of human umbilical cord Wharton's jelly-derived mesenchymal stem cells on lymphocytes. Cell Immunol. 2011;272(1); DOI:10.1016/j.cellimm.2011.09.010.

81. Wang D, Huang S, Yuan X, Liang J, Xu R, Yao G, Feng X, Sun L. The regulation of the Treg/Th17 balance by mesenchymal stem cells in human systemic lupus erythematosus. Cell Mol Immunol. 2017;14(5); DOI:10.1038/cmi.2015.89.

82. Driscoll J, Patel T. The mesenchymal stem cell secretome as an acellular regenerative therapy for liver disease. J Gastroenterol. 2019;54(9); DOI:10.1007/s00535-019-01599-1.

83. Théry C, Witwer KW, Aikawa E, et al. Minimal information for studies of extracellular vesicles 2018 (MISEV2018): a position statement of the International Society for Extracellular Vesicles and update of the MISEV2014 guidelines. J Extracell Vesicles. 2018;7(1); DOI:10.1080/200 13078.2018.1535750.

84. Chudickova M, Vackova I, Machova Urdzikova L, Jancova P, Kekulova K, Rehorova M, Turnovcova K, Jendelova P, Kubinova S. The Effect of Wharton Jelly-Derived Mesenchymal Stromal Cells and Their Conditioned Media in the Treatment of a Rat Spinal Cord Injury. Int J Mol Sci. 2019;20(18); DOI:10.3390/ijms20184516.

85. Giacoppo S, Thangavelu SR, Diomede F, Bramanti P, Conti P, Trubiani O, Mazzon E. Anti-inflammatory effects of hypoxia-preconditioned human periodontal ligament cell secretome in an experimental model of multiple sclerosis: a key role of IL-37. FASEB J. 2017;31(12); DOI:10.1096/ fj.201700524R.

86. Pouya S, Heidari M, Baghaei K, Asadzadeh Aghdaei H, Moradi A, Namaki S, Zali MR, Hashemi SM. Study the effects of mesenchymal stem cell conditioned medium injection in mouse model of acute colitis. Int Immunopharmacol. 2018;54; DOI:10.1016/j.intimp.2017.11.001.

87. Katagiri W, Osugi M, Kawai T, Hibi H. First-in-human study and clinical case reports of the alveolar bone regeneration with the secretome from human mesenchymal stem cells. Head Face Med. 2016;12(1); DOI:10.1186/s13005-016-0101-5.

88. Fukuoka H, Suga H. Hair Regeneration Treatment Using Adipose-Derived Stem Cell Conditioned Medium: Follow-up With Trichograms. Eplasty. 2015;15:e10.

89. Lauxmann MA, Santucci NE, Autrán-Gómez AM. The SARS-CoV-2 Coronavirus and the COVID-19 Outbreak. Int braz j urol. 2020;46(suppl 1); DOI:10.1590/s1677-5538.ibju.2020.s101. 